\section{Peripheral blood flow in patients with diabetes mellitus}

Dear Sir,

The recent paper by Gilmore et al. [1] dealing with "peripheral vasoconstrictor responses and cardiovascular antonomic function tests in diabetic patients" is notable for several reasons: (1) There is no information about the type of diabetes mellitus or the treatment received by the patients. From Table 1 it appears that both insulindependent and insulin-independent patients may have been lumped together, which is a dubious procedure. (2) There is no information regarding glycaemic control at the time of the study, or the relation between treatment and measurement times. (3) Assessment of "central autonomic function" was on the basis of heart rate responses to the Valsalva manoeuvre, deep breathing and standing. Elsewhere [2-4] it has been pointed out how such estimates can produce spurious results.

Apart from these points the paper of Gilmore et al. [1] is particularly remarkable because, although it deals with variability in hand and foot blood flows and responses to cooling, it fails to cite an earlier more extensive, and carefully - controlled study investigating the same topic [5]. Scott et al. [5] carried out detailed cardiovascular testing in well-characterized patients with insulin-dependent diabetes mellitus and, prior to investigating hand, forearm, calf, and foot blood flows (simultaneously), they monitored and managed patients throughout the preceding night to ensure they were relatively euglycaemic before measurements were begun. Apart from peripheral blood flows, measurements were made of systemic arterial blood pressures, heart rate, core temperature and heat production during cooling with a liquid conditioned suit (ie, a procedure identical to that subsequently used by Gilmore et al. [1]. Where there is sufficient information provided in the paper by Gilmore et al. [1] it is clear their results corroborate the findings of Scott et al. [5], and hence failure to cite this publication is misleading for readers unfamiliar with this area of research.

Yours sincerely,

T.Bennett

\section{References}

1. Gilmore JE, Allen JA, Hayes JR (1990) A comparison of peripheral vascoconstrictor responses and cardiovascular autonomic function tests in diabetic patients. Diabetologia 33: $350-356$

2. Bennett T, Farquhar IK, Hosking DJ, Hampton JR (1978) Assessment of methods for estimating autonomic nervous control of the heart in patients with diabetes mellitus. Diabetes 27: 1167-1174

3. Bennett T (1983) Physiological investigation of diabetic autonomic neuropathy. In: Bannister R (ed) Autonomic failure. Oxford University Press, Oxford, pp 406-436

4. Bennett T, Gardiner SM (1988) Cardiovascular pathophysiology in diabetes mellitus: a reappraisal. In: Bannister $R$ (ed) Autonomic failure. Oxford University Press, Oxford, pp 654-666

5. Scott AR, Macdonald IA, Bennett T, Tattersall RB (1988) Abnormal thermoregulation in diabetic autonomic neuropathy. Diabetes 37: 961-968

Prof. T. Bennett

Department of Physiology and Pharmacology

Medical School

Queen's Medical Centre

Nottingham NG7 2UH

UK

\section{Tests of autonomic function in diabetic subjects}

Dear Sir,

We are grateful to Professor Bennett for his comments and take this opportunity of clarifying the points he raises about our recent paper [1]. Our long-term aim was to establish a relatively simple but reliable screening test for cardiovascular autonomic neuropathy in diabetic patients. Accordingly our techniques had to be non-invasive and easily tolerated by the patients. The measurements were carried out in thermally controlled conditions which were thermoneutral for patients and control subjects.

Fifty-two diabetic patients were studied of whom 27 had insulindependent diabetes and 25 had non-insulin-dependent diabetes. The aim of the study was to quantitate autonomic neuropathy which is a well recognised complication of both forms of the disease.

Patients were normally studied in the morning and measurements were commenced at least $3 \mathrm{~h}$ after hypoglycaemic medication had been taken. The mean glycosylated haemoglobin $\left(\mathrm{Hb} \mathrm{A}_{1}\right)$ level in the diabetic patients was 10.2 with a range of 6.3-17.6.

It was appreciated that a single test of heart rate responses may not accurately reflect "central autonomic function" and for this reason the results of three different tests were incorporated in the scoring system described. The tests followed procedures described by Ewing [2] who felt that they have been proved to be of considerable diagnostic use in relating autonomic abnormalities to symptoms and prognosis.

We agree that impairment of thermoregulation may be a consequence of disordered peripheral vascular function in diabetic autonomic neuropathy as previously reported by Scott et al. [3]. The prime purpose of our study however, was to establish an objective assessment of sympathetic regulation of the circulation in the peripheries of diabetic patients. This allows a more complete evaluation of cardiovascular autonomic function than relying solely on tests of reflex heart rate responses.

Yours sincerely,

J.E. Gilmore, J. A. Allen and J. R. Hayes

\section{References}

1. Gilmore JE, Allen JA, Hayes JR (1990) A comparison of peripheral vasoconstrictor responses and cardiovascular autonomic function tests in diabetic patients. Diabetologia 33: 350-356

2. Ewing DJ (1988) Recent advances in the non-invasive investigation of diabetic autonomic neuropathy. In: Bannister R (ed) Autonomic failure. Oxford University Press, Oxford, pp 667-689

3. Scott AR, MacDonald IA, Bennett T, Tattersall RB (1988) Abnormal thermoregulation in diabetic autonomic neuropathy. Diabetes 37: 961-968

Dr. J.E. Gilmore

School of Basic Medical Sciences

The Queen's University of Belfast

97 Lisburn Road

Belfast BT97Bh

Northern Ireland

\section{Causal inference in diabetic nephropathy}

Dear Sir,

Nørgaard et al. [1] state "Our hypothesis is that the rise in blood pressure in Type 1 diabetic patients with renal complications is a consequence of, and not a cause of, nephropathy". This may or may not be true, but their study fails to test the hypothesis. 
In this study, the prevalence of hypertension in the general population is compared to the prevalence of hypertension in groups of diabetic patients. The difference in prevalence of hypertension between the general and the diabetic populations is accounted for by patients with nephropathy because the prevalence of hypertension in patients without nephropathy is similar to that in the general population.

There are several reasons why this observation does not support (or refute) the authors' hypothesis. First, the diabetic groups are either defined in accordance with the authors' hypothesis, or assumed to fit with it. Thus, essential hypertension, which the authors believe does not cause nephropathy, is defined as long-standing hypertension in patients with normal urinary albumin excretion. Clearly neither hypertension nor any other exposure had caused nephropathy in these patients (although hypertension may have been prevented from doing so by treatment). In patients with coexisting nephropathy and hypertension, the temporal relationship between a rise in blood pressure and a rise in urinary albumin excretion - which is the crux of the hypothesis - is not known and cannot be examined because this is a cross-sectional study. That is precisely why it is unwise to infer causation (or lack of it) from cross-sectional studies. Another problem is the use of the general population as a comparison group (intended, I think, to provide a measure of the expected prevalence of non-causal exposure i.e. essential hypertension). People in the general population do not develop diabetic nephropathy because they do not have diabetes; the prevalence of hypertension in this population is irrelevant to the question of whether or not hypertension causes nephropathy in diabetic patients. (In epidemiological terms, the study ignores the effect modification of diabetes on any relationship between hypertension and renal disease).

The hypothesis that hypertension is a consequence of, not a cause of, nephropathy can only be tested by a prospective study which compares the incidence of nephropathy in a group of hypertensive diabetic subjects (exposed) with the incidence of nephropathy in normotensive (unexposed) diabetic patients. The authors' hypothesis would be supported if the incidence of nephropathy were the same in the exposed and unexposed groups (after adjusting for any confounding factors such as age).

However, the argument about whether or not hypertension causes diabetic nephropathy may be oversimplified. The concept of sufficient and component causes [2] recognises that the cause of any disease or complication consists of a constellation of components that act in concert to produce a sufficient cause resulting in disease. If hypertension is causally related to diabetic nephropathy, it will only appear to be so in patients who are also effectively exposed to all other component causes.

In a cross-sectional study of this kind, it would have been more interesting to have seen a comparison of the distribution of absolute blood pressures in diabetic and non-diabetic populations and the relationship between blood pressure and urinary albumin excretion in the diabetic population.

Yours sincerely,

J.Stephenson

\section{References}

1. Nørgaard K, Feldt-Rasmussen B, Borch-Johnsen K, Saelan H, Deckert T (1990) Prevalence of hypertension in Type 1 (insulindependent) diabetes mellitus. Diabetologia 33: 407-410

2. Rothman KJ (1986) Modern Epidemiology. Little, Brown and Company, Boston Toronto, pp 10-16

Dr. J. Stephenson

University College and Middlesex

School of Medicine

Department of Community Medicine

66-72 Gower Street

London WCIE 6EA

UK

\section{Prevalence of hypertension in Type 1 (insulin-dependent) diabetes mellitus}

Dear Sir,

Dr. Nørgaard et al. in their report on the prevalence of hypertension in Type 1 (insulin-dependent) diabetic patients [1] claim that the excess prevalence of arterial hypertension in Type 1 diabetic patients is the result of either incipient or clinical nephropathy while "essential hypertension" is as common as in the non-diabetic population. They conclude that hypertension is unlikely to be a contributory factor to diabetic kidney disease.

Their claim is based on the assumption that essential hypertension is associated with normal urinary albumin excretion. This assumption, however, is not supported by a number of reports [2-4] which have shown increased urinary excretion of albumin in patients with essential hypertension.

We have determined urinary albumin excretion rate in three consecutive overnight collections one week after withdrawal of antihypertensive treatment in the last 16 (11 male, 5 female) patients with essential hypertension attending our outpatient clinic. Their age ranged between 19 and 44 years (mean 31 years), duration of hypertension from 1 to 10 years (mean 4 years), mean \pm SD body mass index was $22 \pm 1.8 \mathrm{~kg} / \mathrm{m}^{2}$, systolic blood pressure $163 \pm$ $18 \mathrm{~mm} \mathrm{Hg}$, diastolic blood pressure $100 \pm 9$ and glomerular filtration rate (measured by ${ }^{51} \mathrm{Cr}$-EDTA clearance) was $113 \pm 7 \mathrm{ml} \cdot \mathrm{min}^{-1}$ $\cdot 1.73 \mathrm{~m}^{2-1}$. All secondary causes of hypertension were ruled out by a full medical work-up and the patients were free from renal, liver and endocrine disease. Diabetes mellitus was excluded by a $75 \mathrm{~g}$ oral glucose load. Figure 1 shows the distribution of albumin excretion rate (median of three determinations) in these patients. Thirty-eight percent were microalbuminuric. There was a significant relationship between mean blood pressure and albumin excretion rate $(r=0.67$; $p<0.01$ ). Thus, microalbuminuria is a feature of "essential hypertension" and Dr. Nørgaard et al. may have overestimated the prevalence of "renal hypertension" in Type 1 diabetic patients by as much as $40 \%$. It is possible that the contribution of essential hypertension to the proteinuria of diabetes is even greater given the increased permeability of capillary vessels in diabetes $[5,6]$.

Moreover diabetes, a salt retaining state $[7,8]$, may facilitate the phenotypic expression of a predisposition, genetic or familial, to essential hypertension. The development of hypertension in a diabetic patient can thus lead to increased protein excretion and subsequent renal damage. The conclusions of Dr. Nørgaard et al. are based on the incorrect premise that essential hypertension has no effect on urinary protein excretion.

The available evidence leads us to suggest that the microalbuminuria of diabetes could well be, in a substantial number of cases, a consequence or a concomitant manifestation of raised blood pressure. It is unlikely that elevated blood pressure is to be ascribed to microalbuminuria (i. e. incipient nephropathy) when glomerular fil-

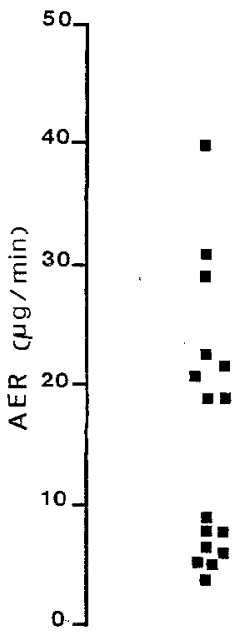

Fig. 1. Urinary albumin excretion rate (AER) in 16 patients with essential hypertension 